\title{
Sensibilización hacia la discapacidad a través de un programa integrado de Educación Deportiva y Aprendizaje-Servicio \\ Disability awareness through an integrated program of sport education and service-learning
}

\author{
Jorge Abellán, Yessica Segovia, David Gutiérrez, Luis Miguel García-López
}

Universidad de Castilla-La Mancha (España)

\begin{abstract}
Resumen. Lograr la aceptación de sus compañeros es una de las estrategias exitosas para favorecer la inclusión del alumnado con necesidades educativas especiales en el aula. La Educación Física (EF) es una de las áreas que ofrece más oportunidades de inclusión. Los objetivos de este trabajo son: (1) presentar una propuesta didáctica que tiene como meta sensibilizar hacia la discapacidad utilizando la integración del Aprendizaje-Servicio (ApS) en el modelo de Educación Deportiva (MED) mediante un deporte adaptado (voleibol sentado); (2) evaluar la percepción del profesorado participante; (3) ofrecer propuestas de mejora para futuras implementaciones. Participaron 181 estudiantes de tercer curso de Educación Secundaria $(E S)(n=80)$ y de quinto curso de Educación Primaria $(E P)(n=101)$. El programa consistió en la creación de dos redes deportivas formadas por dos grupos naturales de ES y otros dos de EP en cada una de las redes. Todos los alumnos vivenciaron en EF una temporada MED a la que se añadieron tres acciones ApS celebradas durante los recreos, siendo los alumnos de ES los emisores del servicio. La unión de los integrantes de la red se creó mediante los elementos distintivos del MED. La sensibilización se realizó a través de la simulación de la discapacidad. Los resultados mostraron una percepción positiva del profesorado, quienes destacaron, además de otros beneficios, el desarrollo de la sensibilización en el alumnado de EP y de la empatía en el de ES. Se discuten las barreras y facilitadores detectados, así como se destacan aspectos clave para potenciar los objetivos educativos.
\end{abstract}

Palabras clave: educación física, modelos pedagógicos, inclusión, diversidad, deporte adaptado.

Abstract. Achieving acceptance by peers is one of the successful strategies to stimulate the inclusion of students with special educational needs (SEN) within their class group. Physical Education is one of the areas that offers more opportunities of inclusion. The objectives of this paper are (1) present a didactic proposal that aims to raise awareness about disability through the integration of Service-Learning (S-L) in the Sport Education Model (SEM) using an adapted sport (sitting volleyball); (2) evaluate the perception of the participant teachers; (3) offer improvement proposals for future implementations. 181 students from the third year of Secondary Education $(\mathrm{SE})(n=80)$ and from the fifth year of Primary Education $(\mathrm{PE})(n=$ 101) participated. The program consisted of the creation of two sports networks formed by two natural groups from SE and two from PE in each of the networks. All the students experienced a SE season at Physical Education in which three S-L actions during recesses were added, with SE students being the service providers. Network members affiliation was promoted through SE characteristics. Awareness was integrated through the simulation of physical disability. The results showed a positive perception of the teachers who highlighted, beside to other benefits, the development of awareness in PE students and of empathy in SE students. Barriers and facilitators are discussed, as well as key aspects to enhance pedagogical goals.

Key words: physical education, pedagogical models, inclusion, diversity, adapted sport.

\section{Introducción}

Durante el curso 2019-2020, un total de 223.805 alumnos recibieron apoyo educativo al ser catalogados como alumnos con necesidades educativas especiales (NEE). En este grupo está incluido el alumnado que requiere de ese apoyo debido a una discapacidad o trastorno grave. De ellos, 185.737 estuvieron

Fecha recepción: 23-12-20. Fecha de aceptación: 21-07-21

Yessica Segovia

yessica.segovia@uclm.es escolarizados en centros ordinarios. Los grupos más numerosos se concentran en Educación Primaria (EP): 83.935 y Educación Secundaria Obligatoria (ES): 59.428 (Ministerio de Educación y Formación Profesional, 2021). En este contexto, la inclusión del alumnado con NEE es un reto para el profesorado de todos los niveles educativos. En el caso del profesorado de Educación Física (EF), los profesores suelen declararse a favor de la inclusión, sin embargo, no se perciben suficientemente preparados para llevarla a cabo con éxito, ya que, entre otros aspectos, se reconocen faltos de herramientas para el diseño de juegos y actividades adaptadas (Reina, Santana, Montesdeoca \& Roldán, 2019). 
De acuerdo con Haegele (2019), la clave podría estar en superar la integración, entendida como el emplazamiento conjunto de alumnos con y sin NEE, y llegar a la inclusión, entendida como una filosofía educativa que promueve la aceptación y valoración por parte del grupo-clase. Entre los aspectos que favorecen la inclusión del alumnado con NEE destacamos la aceptación de sus compañeros, de acuerdo con la propuesta de facilitadores realizada por Wilhelmsen y Sørensen (2019).

Los modelos pedagógicos suponen una práctica educativa en gran auge en EF, y en palabras de Fernández-Río, Hortigüela y Pérez-Pueyo (2018) estos modelos han llegado para quedarse. Sin embargo, en nuestra opinión, uno de los aspectos en los que dicha práctica basada en modelos debería mejorar es en la forma en la que estos modelos pueden (y deben) favorecer la inclusión del alumnado con NEE en las clases ordinarias. La mayoría de los modelos en EF se han diseñado sin tener al alumnado con NEE en mente (Block, Haegele, Kelly \& Obrusnikova, 2020), por lo que son necesarias propuestas que exploren el potencial educativo de los modelos pedagógicos para contribuir a mejorar la atención al alumnado con NEE.

Los objetivos del presente trabajo son: mostrar una propuesta didáctica dirigida a sensibilizar hacia la discapacidad a alumnos de EP y de ES, utilizando una unidad didáctica desarrollada mediante el modelo de Educación Deportiva (MED) que integra acciones de Aprendizaje-Servicio (ApS) y la práctica de un deporte adaptado, el voleibol sentado; evaluar la percepción del profesorado participante, $\mathrm{y}$ ofrecer propuestas de mejora en futuras implementaciones.

\section{Sensibilización hacia la discapacidad en Educación Física}

El posicionamiento teórico de este trabajo se fundamenta a partir de la teoría del comportamiento planificado (TPB, Ajzen, 1991). Las actitudes, entendidas como una idea con carga emocional que nos predispone a actuar de una determinada forma en una situación social concreta (Sherrill, 2004), están influenciadas por las creencias, la experiencia y la información nueva (Kowalski \& Rizzo, 1996). En este planteamiento, las actitudes no se consideran inamovibles, sino que son proclives al cambio, por lo que, proporcionar información y experiencias nuevas pueden modificarlas. Entre las estrategias que se suelen utilizar para ofrecer experiencias nuevas podemos encontrar el contacto directo, en aplicación de la teoría del contacto $(\mathrm{CD}$,
Allport, 1954); y la simulación de la discapacidad, en aplicación de la pedagogía encarnada en un contexto de EF. Como indican Nguyen y Larson (2015) la EF está preparada para introducir la simulación. La finalidad de dicha simulación estaría centrada, entre otros aspectos, en desarrollar la empatía del alumnado participante (Valencia-Peris, Mínguez-Alfaro y Martos-García, 2020). Si la equiparamos a los juegos sensibilizadores, su objetivo sería que los participantes experimenten de forma lúdica las limitaciones a las que se enfrenta una persona con discapacidad con el propósito final de valorar sus capacidades (Ríos, 1994).

Dos recientes trabajos que utilizan la simulación con el objetivo de mejorar las actitudes hacia la discapacidad en EF son: el estudio de Sparkes, Martos y Maher (2019), en el que se examinan las percepciones de dos grupos de futuros maestros al simular osteogénesis imperfecta durante sus clases de EF, incluyendo el primer grupo a una persona con dichao trastorno; y el trabajo de Reina, Iñiguez-Santiago, Ferriz-Morrell, Martínez-Galindo, Cebrián-Sánchez y Roldán (2020) en el que exploran los diferentes efectos de programas de cambio de actitudes, modificando su duración, contacto y estrategias utilizadas.

En general, «el uso de simulaciones se antoja como una óptima herramienta para mejorar las actitudes hacia la inclusión» (Valencia-Peris et al., 2020, p. 601), aunque, de acuerdo con Smith (2008), tendremos que afirmar que la simulación no es una herramienta infalible, y se debe utilizar siempre que se asuman sus límites.

\section{Educación deportiva}

El MED (Siedentop, 1994) es un modelo pedagógico que nació por y para la EF, con la principal motivación de ofrecer la oportunidad a todo el alumnado de vivir una experiencia deportiva auténtica (Siedentop, 2002) a partir del diseño e implementación de unidades didácticas de larga duración (entre 12-20 sesiones), denominadas temporadas, en las que se le otorga al alumnado responsabilidad (Siedentop, Hastie, \& van der Mars, 2020).

El diseño de estas temporadas se caracteriza por reproducir en la clase de EF aquellas características del deporte institucional que lo hacen tan especial y motivante para sus practicantes (Siedentop, 1994): (1) estructuración de los contenidos siguiendo las fases de una temporada deportiva (p.ej., pretemporada, fase regular, fase final); (2) afiliación durante toda la temporada a un mismo grupo de trabajo (equipo); (3) establecer un calendario de competición; (4) registro 
de datos o estadísticas, tanto relacionadas con el rendimiento colectivo e individual como otros resultados que tengan en cuenta el juego limpio, el desarrollo de responsabilidades, etc.; (5) fomentar un clima festivo (colores de equipos, cánticos, mascota, banderas, himno de la competición, cartelería...); y por último, (6) ofrecer un evento culminante, donde, en un ambiente de festividad, se culmina tanto el proceso de enseñanzaaprendizaje como el desarrollo de la temporada deportiva (García-López \& Gutiérrez, 2016).

Por otro lado, en la búsqueda de evitar reproducir los errores del deporte institucional, y maximizar los logros educativos, se introducen dos estrategias que potencian el carácter educativo del modelo (GarcíaLópez \& Gutiérrez, 2016). La primera de ellas es el desarrollo de responsabilidades a través de roles, que permite una mayor vivencia y conocimiento de la cultura deportiva. Para ello, se asigna a cada alumno dos roles, el rol de jugador más un rol complementario que desarrolla responsabilidades de equipo o de organización (p.ej., entrenador, árbitro, preparador físico, reportero, etc.). En segundo lugar, se adapta el contenido a las características evolutivas de los participantes, al mismo tiempo que se busca una máxima participación de todo el alumnado.

La suma de los seis elementos deportivos y las dos adaptaciones educativas potencian el desarrollo deportivo integral de los estudiantes (García-López y Gutiérrez, 2016) concretándose en la triple meta de enseñar a los alumnos a ser deportistas (1) competentes, (2) cultos y (3) entusiastas (Siedentop, 1994). El MED, pudiendo considerarse como uno de los modelos pedagógicos más populares e investigados (Hastie, Martínez de Ojeda \& Calderón, 2011; Wallhead \& O'Sullivan, 2005), ha mostrado un gran potencial educativo en cada uno de los dominios de aprendizaje (Evangelio, Sierra-Díaz, González-Víllora, \& FernándezRío, 2018; Guijarro, Rocamora, Evangelio, GonzálezVillora, 2020), con especial atención al dominio social en aspectos tales como el desarrollo ético (Harvey, Kirk, \& O'Donoven, 2014) o el desarrollo de habilidades personales y sociales (Bessa, Hastie, Araújo \& Mesquita, 2019).

La plasticidad de este modelo permite potenciar objetivos educativos incluyendo estrategias educativas adicionales a sus características. En los últimos años, destaca la combinación o hibridación de modelos pedagógicos como una de las estrategias más populares, que permite potenciar los beneficios educativos que aportan la unión de los modelos. Esta tendencia fue estudiada por González-Víllora, Evangelio, Sierra-Díaz y Fernández-Río (2019) mostrando que el MED es el modelo más hibridado, en especial con modelos basados en el juego. Recientemente, se ha buscado enriquecer y potenciar el MED con estrategias metodológicas de carácter social, como el ApS. En concreto, García-López, Gutiérrez y Fernández-Bustos (2019) muestran una propuesta de integración del ApS en el MED. Esta combinación también se ha utilizado en la presente propuesta didáctica.

\section{Aprendizaje-Servicio}

El ApS es considerado por Chiva-Bartoll y Fernández-Río (2021, p. 11) como un «modelo pedagógico de carácter activista, transformador, transdominio e intercontextual» en el que los estudiantes ponen en práctica contenidos curriculares durante la participación activa en trabajos comunitarios (GarcíaLópez et al., 2019). Basándonos en su definición, y de acuerdo con Bringle y Hatcher (1999), a diferencia de otros servicios comunitarios en los que se pueden obtener beneficios educativos en su realización, en el ApS las actividades y sus respectivos objetivos educativos son integrados de forma intencionada. La necesidad cubierta por estos servicios (denominada trabajos comunitarios especialmente en el entorno universitario) debe lograr conseguir los objetivos curriculares, a la vez que son actividades significativas para ambos colectivos (Bringle y Hatcher, 1999). Por tanto, el servicio a la comunidad y un aprendizaje planificado son los dos ejes básicos de esta metodología. Además, para que se dé un aprendizaje significativo, debe plantearse un proceso de aprendizaje, reflexión y acción (PuigRovira y Palos-Rodríguez, 2006). Así, una parte importante de esta metodología es la estrategia de reflexión, pues tal y como exponen Bringle y Hatcher (1999), los trabajos realizados para la comunidad no tienen por qué producir aprendizaje por sí mismos en los estudiantes, mientras que las actividades reflexivas permiten dirigir el aprendizaje uniendo el servicio prestado y el contenido curricular.

El ApS podría ser un modelo adecuado para el trabajo de habilidades sociales, y en concreto de la sensibilización, pues estudios y experiencias llevadas a cabo con universitarios han mostrado el potencial del trabajo de este dominio. Por ejemplo, Chiva-Bartoll, MaravéVivas, Salvador-García y Valverde-Esteve (2021) utilizaron un enfoque mixto para analizar el efecto de un programa de ApS dirigido a niños con trastorno del espectro autista y llevado a cabo por futuros maestros 
de EF. Entre los resultados de los prestadores del servicio destaca la idea de que el programa ha ayudado a su crecimiento personal y les ha proporcionado una formación inicial de calidad. En este sentido, con esta orientación de trabajo se cumpliría con la idea de Capella, Gil y Martí (2014) en la que las actividades de ApS deberían estar orientadas hacia la educación en valores, en este caso orientado hacia la sensibilización hacia la discapacidad.

A pesar de que el ApS no es una metodología específica del área de EF, en los últimos años su aplicación e investigación ha incrementado considerablemente. Tal es así, que se pueden consultar diversas revisiones sistemáticas en el contexto de la EF y/o la actividad física (p.ej., Carson y Raguse, 2014; Chiva-Bartoll et al., 2019; Pérez-Ordas, Nuviala, Grao-Crucesy Fernández-Martínez, 2021). Sin embargo, sigue habiendo pocas investigaciones que integren el ApS en EF en edad escolar (p.ej., Calvo, Sotelino, \& Rodríguez, 2019) en comparación con la introducción de esta metodología en la educación superior. Por lo tanto, en este artículo se presenta una perspectiva novel de trabajo, con la aplicación del ApS en un contexto de EPES y con una orientación hacia la educación en valores.

\section{Deporte adaptado}

Una de las formas habituales de simular una discapacidad en EF es utilizar la práctica de un deporte adaptado. Un deporte adaptado es aquel que se adecúa a las características específicas de los participantes, personas con discapacidad. Los deportes adaptados pueden clasificarse en tres tipos: aquellos que se adaptan a partir de un deporte normalizado, como por ejemplo el baloncesto en silla de ruedas; los que son específicos, como el goalball; y los que no incluyen ninguna modificación, como el atletismo para personas con discapacidad intelectual.

Los deportes adaptados pueden cumplir una triple función en la didáctica de la EF escolar: como un contenido propio de EF, como un juego sensibilizador y como un deporte alternativo (Abellán \& Sáez-Gallego, 2017). En este trabajo se presenta una propuesta didáctica que trata de hacer cumplir esa triple perspectiva:

Contenido propio de EF: utilizando el deporte adaptado como contenido de iniciación deportiva dentro del MED, que integra ApS, de acuerdo con la propuesta de García-López et al. (2019).

Juego sensibilizador: como experimentación de las limitaciones y valoración de capacidades de una persona con discapacidad, de acuerdo con la definición de juego sensibilizador de Ríos (1994).

- Deporte alternativo: mejorando el disfrute y la motivación del alumnado debido a la práctica de deportes no practicados habitualmente.

En algunos trabajos previos se han utilizado diferentes tipos de deportes adaptados dentro de los programas de cambio de actitudes hacia la discapacidad. Por ejemplo, Pérez-Torralba, Reina, Pastor-Vicedo y GonzálezVíllora (2018) emplearon dos deportes adaptados (boccia y goalball), diseñados específicamente para poblaciones con grandes necesidades de apoyo, en un programa de cambio de actitudes hacia la discapacidad en EF en EP. Los resultados del programa indicaron que las actitudes del grupo experimental mejoraron significativamente después de la realización de la intervención.

Mas allá de la utilización de los deportes adaptados con el objetivo de mejorar las actitudes hacia la discapacidad, son pocos los trabajos que los han tratado como un contenido más de EF y por tanto susceptible de una orientación basada en la obtención de mejoras educativas. En este sentido, Robles-Rodríguez, AbadRobles, Fuentes-Guerra y Benito-Peinado (2017) evaluaron la opinión del alumnado de Bachillerato sobre la contribución de los deportes adaptados como contenido de EF. Los resultados mostraron que el alumnado percibió que la práctica de deportes adaptados, en contraposición con los deportes tradicionales, suponía una contribución a su educación en valores y sensibilización social, sin embargo, los resultados mostraron que eran los deportes tradicionales los que favorecían más al desarrollo de habilidades y destrezas motrices.

Para la presente propuesta se ha elegido el voleibol sentado, un deporte de cancha dividida y participación alternativa en el que dos equipos tratan de que el equipo contrario no pueda devolver un balón enviado a su campo. Con el objetivo de que el alumnado simulara tener discapacidad física, y como regla propia del deporte, los estudiantes debían permanecer sentados durante todo el desarrollo de todas las sesiones. El voleibol sentado es un deporte paralímpico en el que se enfrentan dos equipos de seis jugadores, con las mismas reglas que el voleibol y con adaptaciones destinadas a la participación de personas con discapacidad física (i.e. altura de la red, tamaño del campo, etc.).

\section{Justificación}

Llegados a este punto cabe preguntarse si el programa de sensibilización utilizando el deporte adaptado podría 
ser más efectivo si se combinara con otros modelos o metodologías de EF que han demostrado su éxito educativo, para ser más concretos: ¿Se podrían maximizar sus efectos utilizándolo de manera combinada con otros modelos de éxito? En este sentido, Menéndez y Fernandez-Río (2017) mostraron la eficacia de un programa hibrido entre MED y Modelo de responsabilidad personal y social en la inclusión de alumnos con discapacidad. Desde el conocimiento de los autores, no existen propuestas que combinen la utilización del MED, con ApS integrado, en un programa de cambio de actitudes hacia la discapacidad utilizando un deporte adaptado.

En la Figura 1 se presenta la relación entre los aspectos más importante que presenta esta propuesta didáctica a partir de las aportaciones de cada una de las metodologías empleadas. Los aspectos compartidos por el MED y el ApS se centrarían en la asunción de roles y en la responsabilidad otorgada a los estudiantes. Los aspectos compartidos entre el MED y el programa de sensibilización se refieren al propio contenido deportivo, en este caso el voleibol sentado, y con la simulación como estrategia de sensibilización, que surge de la propia práctica del deporte $\mathrm{y}$, finalmente, los aspectos compartidos entre el ApS y la sensibilización tendrían que ver con la educación en valores, especialmente con el trabajo de la empatía.

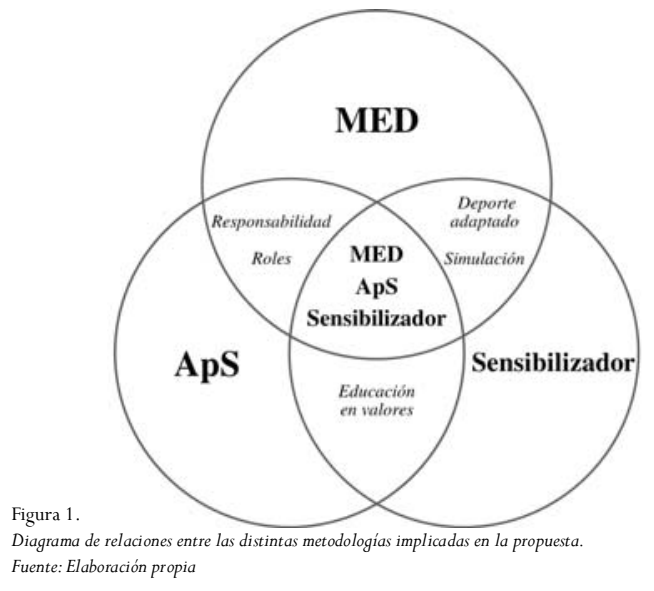

¿Podría el deporte adaptado aumentar su potencial sensibilizador a través de su utilización combinada con el MED y el ApS? Con el objetivo de responder a esta cuestión, a continuación, se desarrolla una propuesta práctica que combina el MED y el ApS utilizando como contenido educativo el deporte adaptado del voleibol sentado y con el objetivo fundamental de mejorar las actitudes hacia la discapacidad del alumnado participante, utilizando para ello la simulación de la discapacidad física.

\section{Planteamiento didáctico}

\section{Participantes y contexto}

En esta propuesta didáctica participaron 181 estudiantes de tercer curso de ES y de quinto curso de EP de un centro de enseñanza concertado de una localidad del centro de Castilla-La Mancha (España), pertenecientes a ocho grupos naturales. Las características de los participantes se muestran en la Tabla 1 .

\begin{tabular}{lccc}
$\begin{array}{l}\text { Tabla 1. } \\
\text { Caracteristicas de la muestra }\end{array}$ & \multicolumn{3}{c}{} \\
\hline \multicolumn{5}{c}{ Muestra total } & $3^{\circ} \mathrm{ESO}$ & $5^{\circ} \mathrm{EP}$ \\
& $N(M \pm D T)$ & $n(M \pm D T)$ & $n(M \pm D T)$ \\
\hline Total & $181(11.86 \pm 2.05)$ & $80(14.06 \pm .62)$ & $101(10.12 \pm .57)$ \\
Chicos & $84(12.23 \pm 2.11)$ & $45(14.09 \pm .67)$ & $39(10.08 \pm .62)$ \\
Chicas & $97(11.55 \pm 1.95)$ & $35(14.03 \pm .57)$ & $62(10.15 \pm .54)$ \\
\hline
\end{tabular}

Ambos grupos de estudiantes, emisores y receptores participaron en un programa de sensibilización hacia la discapacidad en sus clases de EF. Sin embargo, con la meta de profundizar más ante la necesidad detectada en la población objeto de estudio de sensibilizar hacia la discapacidad, se integraron en el programa de EF acciones ApS. En estas acciones ApS, los estudiantes de ES tuvieron el rol de emisores del servicio, mientras que los alumnos de EP recibieron el servicio. Debido a que todos los grupos pertenecían al mismo centro escolar, las acciones de ApS se llevaron a cabo en horario escolar durante los recreos y clases de EF de los alumnos que recibían el servicio (horario establecido para los estudiantes de EP).

Respecto a los profesores que desarrollaron el programa, se trataba de dos hombres, uno de ellos se encargaba de la docencia en los grupos de EP (41 años, 10 años de experiencia docente) y el otro de la docencia en ES (39 años, nueve años de experiencia docente).

\section{Procedimiento}

El presente planteamiento didáctico pertenece a una investigación más amplia (Proyecto: Impacto del modelo Educación Deportiva como Aprendizaje-Servicio en el desarrollo de la competencia social y cívica. EDU201786928-R) que contempló cuatro fases. En la primera fase, los investigadores diseñaron y planificaron el proyecto, incluyéndose en la planificación una experiencia didáctica que combinara el MED y el ApS utilizando un deporte adaptado con el objetivo de sensibilizar hacia la discapacidad en EF. Tras el diseño inicial, los investigadores contactaron con el centro participante a través de su equipo directivo para explicarles el objetivo del estudio. El diseño inicial de la experiencia fue adaptado a las particularidades del 
alumnado del centro, cuyas características específicas se detallan en el siguiente apartado, destinado al programa. Por último, se obtuvieron los consentimientos informados de los padres o tutores legales de los estudiantes y de los docentes. En la segunda fase, se estableció el cronograma de la experiencia de forma conjunta entre los docentes y el equipo de investigación. Además, durante esa fase se estableció un programa de formación para los docentes: curso teórico-práctico de 20 horas del MED, jornadas de formación en ApS de aproximadamente tres horas, y, por último, una reunión con el investigador principal para revisar y explicar el programa de intervención, con especial atención al desarrollo del contenido deportivo (voleibol sentado) con el objetivo de sensibilizar al alumnado. En la tercera fase se implementó el programa de intervención. Por último, se evalúo la primera puesta en práctica del proyecto, utilizando la percepción del profesorado mediante la entrevista, cuyos resultados se muestran en el apartado de resultados del presente trabajo.

\section{Programa}

Se integraron dos elementos clave en el MED para fomentar la sensibilización de los alumnos hacia la discapacidad: metodología ApS y la utilización de un deporte adaptado. La integración del ApS permitió fomentar la vocación hacia el servicio utilizando como principal estrategia la micro-enseñanza de un deporte adaptado, es decir, el alumnado perteneciente a los grupos de ES (emisores del servicio) enseñó al alumnado de EP (receptor del servicio). Asimismo, se utilizó como contenido didáctico el voleibol sentado por la capacidad sensibilizadora de los deportes adaptados, y en concreto la técnica la simulación.

Se crearon dos redes deportivas formadas cada una de ellas por dos grupos naturales de tercer curso de ES y dos quintos cursos de EP del mismo centro educativo. Siguiendo los requisitos del MED se planteó una temporada integrada en EF de 14 sesiones desarrollada en cada grupo-clase, a las que se sumaron tres acciones ApS realizadas durante tres recreos (30 minutos por recreo) y dos sesiones de EF en el horario de EP. La distribución de la temporada y las acciones ApS se pueden consultar en laTabla 2. El número de sesiones en EF y su duración dependió de la etapa educativa a la que pertenecían los estudiantes, de tal forma que mientras en EP la EF constaba de tres sesiones de 45 minutos, en ES fueron dos sesiones de 55 minutos.

Para la organización y planificación del MED en EF se utilizó como base la temporada de Ringo de García-
López y Gutiérrez (2016), con una duración de 16 sesiones divididas en las fases de: organización (dos sesiones), pretemporada (seis sesiones), fase regular (seis sesiones) y fase final (dos sesiones). A partir de la planificación inicial, los docentes adaptaron el número de sesiones a cada uno de los grupos debido a las características de su calendario educativo. Finalmente, el número de sesiones de EF dedicadas a la temporada osciló entre las 13 y las 16 sesiones. Las características del MED se utilizaron como punto de unión entre los grupos-clase incluidos en cada una de las redes: (1) clubes con representación en cada uno de los grupo-clase; (2) identidad de equipos (color de camiseta, bandera y nombres) comunes al club; (3) competición intragrupo e intergrupo con registro de datos común para los clubes; y (4) evento final intragrupo e intergrupo.

La tabla 2 muestra un resumen de la temporada tipo. En dicha temporada se integraron las acciones ApS, en las que el alumnado de ES prestó el servicio de formación y dinamización, siendo el alumnado de EP el receptor del servicio. Las acciones ApS, se realizaron a modo de convivencia durante los recreos. Se realizaron dos convivencias y un evento final culminante intergrupo, en el que el alumnado de ES ejerció los roles organizativos (entrenador, preparador físico, anotador, árbitro y periodista) y el de EP el de jugadores. Durante los encuentros, los docentes desarrollaron responsabilidades de supervisión y guía de los alumnos de ES. Las acciones ApS consistieron en la enseñanza de elementos técnico-tácticos del voleibol sentado a través de juegos modificados. Para realizar estas acciones ApS, el alumnado de secundaria se formó previamente en sus clases de EF, consistiendo su formación principalmente en el aprendizaje del contenido y la vivencia en el desarrollo de responsabilidades durante la temporada MED desarrollada en su grupo-clase.

Respecto al programa de sensibilización, se utilizaron los tres niveles sugeridos porWilson y Lieberman (2000) y que Foley, Tindall, Lieberman y Kim (2007) ya habían integrado previamente en una temporada del MED en la que se utilizó un deporte adaptado. El primer nivel, «exposición», trata de dar a conocer a los estudiantes la discapacidad a través del deporte adaptado. En este nivel suelen utilizarse por ejemplo videos de personas con discapacidad practicando el deporte en cuestión. El segundo nivel, «experiencia», incluye el desarrollo de juegos en los que se simule la discapacidad. Finalmente, el tercer nivel, «propiedad», es el más avanzado de los programas de sensibilización, en él los estudiantes pueden profundizar en el tratamiento de la sociedad a 
las personas con discapacidad. De acuerdo con Foley et al. (2007) en este nivel encajan los torneos y competiciones propias del MED. Además, en todo el programa se pueden incluir el trabajo de roles y responsabilidades que ayudan a profundizar en el conocimiento del deporte adaptado y a través de él de las personas con discapacidad que lo practican. De forma específica, en el programa que aquí se presenta se utilizó un video de voleibol sentado para el Nivel 1, la simulación de la discapacidad física durante el desarrollo de la experiencia para el Nivel 2 y la responsabilidad de los roles desempeñados durante la temporada MED para el Nivel 3. Además, el grupo de alumnos de ES tuvo un extra de responsabilidad al tener que desarrollar roles organizativos durante la realización de las acciones ApS.

En cuanto al diseño de progresión de enseñanza de los contenidos deportivos, se emplearon juegos modificados y tareas correctivas, diseñadas y adaptadas a partir del trabajo de enseñanza del voleibol a pie de Sánchez-Gómez (2011) y de la propuesta de ringo de García-López y Gutiérrez (2016). Se utilizaron juegos modificados por representación y/o exageración con el objetivo de trabajar los principios tácticos de los juegos de red y muro. Para ello, se modificaron los diferentes elementos estructurales del juego. A modo de ejemplo: número de jugadores (1vs1, 2vs2, 3vs3 y 4vs4), terreno de juego (diferentes dimensiones de campo y usos: alargados, anchos y cortos) materiales (cambio de móvil: pelota de playa) y reglas (un jugador puede agarrar y lanzar). Como formato de juego base de competición se utilizó el 4 vs. 4, en un terreno de juego adaptado al número de jugadores de aproximadamente de $7 \times 14$ metros, y se usó como móvil una pelota de playa en lugar de los habituales balones de voleibol.

\section{Instrumentos de evaluación y análisis}

Para conocer la percepción del profesorado participante (EP y ES), al finalizar el programa se realizó

\begin{tabular}{|c|c|c|c|c|c|}
\hline \multicolumn{6}{|c|}{ Programa de intervención } \\
\hline Fase & Sesiones EF & $\begin{array}{c}\text { Acciones } \\
\text { ApS }\end{array}$ & Sensibilización & MED & $\mathrm{ApS}$ \\
\hline Organización & $1-2$ & & $\begin{array}{l}\text { Nivel 1: exposición. Estrategia } \\
\text { de la información } \\
\text { (video voleibol sentado) }\end{array}$ & $\begin{array}{l}\text { Motivación y organización de las } \\
\text { características esenciales del } \\
\text { MED (afiliación, roles, } \\
\text { calendario de la temporada) }\end{array}$ & $\begin{array}{l}\text { Motivación hacia la } \\
\text { vocación al servicio } \\
\text { comunitario }\end{array}$ \\
\hline Pretemporada & $3-7$ & 1 recreo & $\begin{array}{l}\text { Nivel 2: experiencia. } \\
\text { Estrategia de simulación. } \\
\text { Nivel 3: propiedad. } \\
\text { Responsabilidad en roles ApS. }\end{array}$ & $\begin{array}{l}\text { Formación en responsabilidades } \\
\text { y elementos técnico-tácticos del } \\
\text { deporte }\end{array}$ & $\begin{array}{l}\text { Recreo 1. Prestación } \\
\text { servicio } \\
\text { (entrenamiento) }\end{array}$ \\
\hline Fase regular & 8-12 & 1 recreo & $\begin{array}{l}\text { Nivel 2: experiencia. } \\
\text { Estrategia de la simulación. } \\
\text { Nivel 3: propiedad. } \\
\text { Responsabilidad en roles ApS. }\end{array}$ & $\begin{array}{l}\text { Práctica autónoma de las } \\
\text { responsabilidades y práctica de } \\
\text { contenidos deportivos en } \\
\text { situaciones de juego reales } \\
\text { (competición) }\end{array}$ & $\begin{array}{l}\text { Recreo 2. Prestación } \\
\text { servicio } \\
\text { (entrenamiento) }\end{array}$ \\
\hline Fase final & $13-14$ & $\begin{array}{l}2 \text { sesiones d } \\
45 \text { minutos } \\
1 \text { recreo }\end{array}$ & $\begin{array}{l}\text { Nivel 3: propiedad. } \\
\text { y Responsabilidad en roles ApS. }\end{array}$ & $\begin{array}{l}\text { Culminación del proceso de } \\
\text { enseñanza-aprendizaje } \\
\text { (contenido y festividad) }\end{array}$ & $\begin{array}{l}\text { Recreo 3. Fase final } \\
\text { clubes }\end{array}$ \\
\hline
\end{tabular}

una entrevista semiestructurada con cada uno de ellos por separado. Las preguntas versaban sobre su opinión sobre el desarrollo del programa y su percepción sobre las actitudes del alumnado.

Las entrevistas fueron transcritas literalmente y analizadas desde un enfoque cualitativo de contenido deductivo, agrupando las respuestas en primer lugar en una categoría de Satisfacción general del programa, y después en función de cada uno de los aspectos del programa: MED, ApS, Sensibilización y Deporte adaptado. Los investigadores utilizaron como apoyo el programa de análisis cualitativo ATLAS.ti Cloud. Para ello, se crearon 10 preguntas que se dividieron en cinco categorías: percepción general del programa (p.ej., ¿Cuál es tu opinión sobre el programa?), sensibilización (p.ej., ¿Cómo crees que se han sentido tus alumnos al simular la discapacidad física?), deporte adaptado (p.ej., ¿Qué te ha parecido la utilización del voleibol sentado como contenido de EF?), MED (p.ej., ¿Has tenido alguna dificultad en la utilización del modelo?) y ApS (p.ej., ¿Cómo crees que han vivido los alumnos las convivencias ApS?).

\section{Resultados}

La tabla 3 muestra el resumen de los hallazgos derivados de la percepción de los docentes.

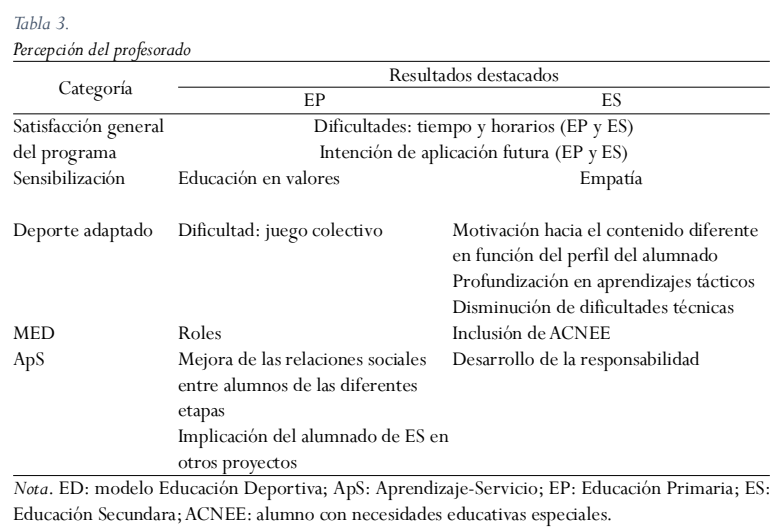

En cuanto a la percepción general del programa, ambos docentes destacaron que el programa les pareció muy interesante. Sin embargo, una de las dificultades percibidas fue «las prisas» (docente de EP), debido a la ubicación temporal en el tercer trimestre del curso, ya que ocasionó que tuvieran poco tiempo para realizar las actividades o que debido a la duración del programa tuviesen que 
pedir horas prestadas a otros compañeros. Ambos docentes declararon que les gustaría seguir realizando la actividad el curso siguiente, intentando tener reuniones para la planificación cerca de la implementación del programa, ya que, como indica el profesor de ES: «nosotros [los dos profesores y el investigador principal] de cara a lo organizativo hemos tenido las reuniones muy salteadas» (docente de ES). Este aspecto se puede entender como una propuesta de mejora en intervenciones futuras.

El aspecto que ambos docentes han destacado por encima del resto ha sido la percepción sobre la sensibilización que se ha producido en sus alumnos realizar el programa. En el caso del docente de EP, relaciona directamente la sensibilización con la educación en valores: «los valores hay que trabajarlos y es una buena manera de trabajarlos, con un deporte adaptado» (docente de EP), mientras que su compañero de ES destaca el trabajo de la empatía: «fomentar esa empatía, ese ponerse en los zapatos del otro... siempre suponen experiencias enriquecedoras para los alumnos» (docente de ES). Ambos creen que el alumnado participante ha podido sentir lo que siente una persona con discapacidad física al practicar voleibol sentado.

Respecto a la utilización del deporte adaptado como contenido de EF, percibieron que el voleibol sentado ha tenido diferentes grados de aceptación en sus alumnos, «había algunos alumnos que estaban enganchados, ya no al programa o la competición, sino al voleibol sentado, les gustaba, pero a otros muchos les ha parecido poco dinámico y un poco aburrido» (docente de ES). Un aspecto muy positivo que han destacado ha sido la posibilidad de que el alumnado realice aprendizajes tácticos, que en muchos casos se pueden transferir a otros deportes de red y muro: «tiene muchas similitudes y mucha transferencia con el voleibol [a pie] en cuanto al reglamento, dinámica de juego...» (docente de ES), además «no ha necesitado mucha técnica, no ha necesitado una gran cantidad de tiempo invertido para dominarlo» (docente de ES). Aunque en la etapa de EP se ha producido una dificultad a la hora de construir juego, ya que: «a pesar de que estaban concienciados y sabían las normas y sabían que tenían hasta la posibilidad de dar tres toques, era tipo jugar al tenis [utilizando un solo golpeo]». Respecto a este último aspecto, el maestro de EP indica una sugerencia para tratar de solucionarlo: «repetir un poco más» (docente de EP). Aunque en este punto de la entrevista finalmente confiesa: «no he seguido a pies juntillas los ejercicios que había aquí [programación entregada por el investigador principal]» (docente de
EP). Por ello, cabe la posibilidad de que este déficit pueda ser imputable a no seguir el programa de manera más fiel, al nivel de desempeño del alumnado de EP, o a una conjunción de ambos aspectos.

En lo referente a la utilización del $M E D$, el docente de EP ha destacado que cada alumno se ha identificado con su rol. Además, parece que el programa ha ayudado a desarrollar el potencial del alumnado con NEE, ya que:

«Un par de alumnos que se encuentran en un programa de PMAR [programa de mejora del aprendizaje y rendimiento para alumnado con NEE], que normalmente en las clases hay que estar un poco tirando de ellos y sin embargo (...) al tener un rol asignado se lo han tomado súper en serio» (docente de ES).

Finalmente, la utilización del $A p S$ ha permitido que mejoren las relaciones del centro entre el alumnado de EP y ES: «los he visto muy integrados y me ha gustado porque había bastantes de secundaría que sí que lo vivían y lo disfrutaban y sé que es una buena manera de mejorar las relaciones entre ellos, de interaccionar» (docente de EP). Además, ha tenido resultado más allá de la propia implementación del programa, como relata el maestro de EP: «estoy con la coreografía y una alumna de ES me ha preparado la coreografía y están los niños... la abrazan cuando viene» (docente de EP). En el caso del alumnado de ES, realizar las acciones ApS los llevó a «demostrar y responsabilizarse de un grupo realmente de niños ejerciendo un papel de mayores» (docente de ES).

\section{Decisiones de acción para la próxima propuesta en práctica}

A partir de los resultados obtenidos de la puesta en práctica de la propuesta didáctica se muestran diferentes sugerencias para futuras implementaciones. Para facilitar su presentación, estas decisiones de acción se presentan a partir de las dificultades detectadas (Tabla 4).

A partir de la puesta en práctica de la propuesta, de Tabla 4

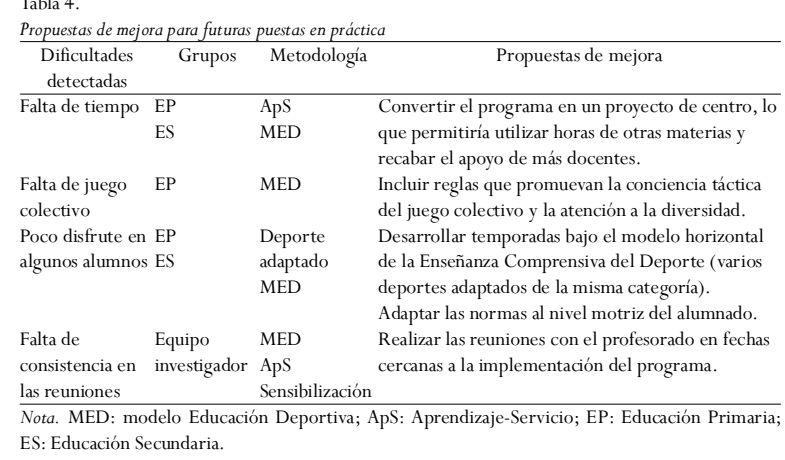


la percepción del profesorado implicado y de la detección de dificultades, se propone que, en futuras intervenciones, se intensifique la atención a los siguientes aspectos clave, que, en opinión de los autores, pueden resultar relevantes para su efectividad educativa:

Un proyecto de esta envergadura requiere de la implicación del centro o centros implicados, y no tener que ceñirse a los (escasos) recursos horarios del área de EF. En este sentido, como se ha mencionado en la Tabla 3 , parece que la conversión de esta intervención en un proyecto de centro podría favorecer la implicación del profesorado del centro y conseguir un carácter interdisciplinar con la aportación de otras áreas. En este sentido, se puede consultar la propuesta de Educación Deportiva por Proyectos de Gutiérrez y Segovia (2017).

- Los programas de sensibilización requieren de una puesta en práctica marcada por la reflexión. La mera práctica de deporte adaptado no consigue calar tan profundo en el alumnado, por lo que el profesorado implicado debe asegurar espacios de reflexión antes y después de la realización de las sesiones. Para ello cabe resaltar la necesidad de aumentar la formación del profesorado en programas de sensibilización y de esta manera igualarlo a la formación recibida en el MED y en la realización del ApS. A este respecto, se sugiere que al terminar las sesiones los docentes preparen un pequeño debate sobre cómo se han sentido los alumnos al simular la discapacidad, tratando de orientar las respuestas hacia la idea de la valoración de capacidades de las personas con discapacidad.

- En relación con el aprendizaje deportivo, con especial atención en el desarrollo del juego colectivo, se recomienda diseñar y aplicar modificaciones de los elementos estructurales del juego. A modo de ejemplo se podrían establecer diferentes puntuaciones en función del número de jugadores implicados (un punto para jugadas que impliquen un solo jugador, dos puntos para jugadas que impliquen dos jugadores, o tres puntos para jugadas que impliquen tres o más jugadores). Igualmente, se deberían incluir modificaciones que atiendan a la diversidad con el objetivo de maximizar la participación y el disfrute de todos los estudiantes. Se sugieren dos modificaciones a modo de ejemplo: limitar la devolución directa con el objetivo de fomentar la fase de colaboración y eliminar la técnica de golpeo pudiendo coger y lanzar el móvil.

- Por último, para aumentar la efectividad del programa, la formación del alumnado que presta el servicio se debería llevar a cabo desde una triple vertiente: conocimiento deportivo, recursos para desarrollar competencias para enseñar (habilidades comunicativas, sociales...) y alto grado de sensibilización hacia la discapacidad que les permita incidir en este aspecto cuando enseñan el deporte a los más pequeños.

\section{Conclusiones}

El principal objetivo por el que se planteó el programa era diseñar una propuesta desde el área de EF dirigida a sensibilizar hacia la discapacidad a alumnos de EP y ES. La evaluación de esta experiencia ha mostrado que los docentes que participaron en la propuesta percibieron positivamente el programa, destacando la sensibilización hacia la discapacidad conseguida en su alumnado a través de la educación en valores, y con especial atención en el desarrollo de la empatía. Asimismo, los docentes percibieron el deporte adaptado como un contenido adecuado para profundizar en los contenidos tácticos a la vez que permite una disminución de las dificultades técnicas, aunque en EP destacaron la dificultad de desarrollar la conciencia del juego colectivo. Por otro lado, y en relación con las metodologías destacaron el desarrollo de competencias sociales como la responsabilidad (MED y ApS), el potencial de inclusión de alumnos con NEE (MED) y la implicación de estudiantes en el desarrollo de la vida escolar (ApS).

La evaluación y seguimiento del programa permitió establecer mejoras para futuras implementaciones del proyecto en relación con las dificultades detectadas por los docentes. Asimismo, se han descrito aspectos clave relacionados con la implicación de la comunidad educativa, la formación docente, el diseño y adaptación del contenido deportivo a las características del alumnado y la formación del alumnado que presta el servicio, que han sido considerados clave por los autores para potenciar los objetivos educativos propuestos en el programa.

En conclusión, la combinación metodológica MEDApS y el uso de deporte adaptado (MED-ApS sensibilizador) parecen tener gran potencial para desarrollar habilidades sociales y personales en el alumnado, que ayudan a potenciar la sensibilización hacia la discapacidad en el área de EF.

\section{Financiación}

Este trabajo se realizó dentro del proyecto: Impacto del modelo Educación Deportiva como Aprendizaje-Servicio en el desarrollo de la competencia social y cívica (EDU201786928-R), financiado por el Ministerio de Ciencia e 
Innovación -Agencia Estatal de Investigación y los fondos FEDER. Yessica Segovia es beneficiaria de un contrato predoctoral para la formación de personal investigador en el marco del Plan Propio de I+D+i, (2018CPUCLM-7487) susceptible de cofinanciación por el fondo Social Europeo [2018/12504] en la Universidad de Castilla-La Mancha.

\section{Referencias}

Abellán, J., \& Sáez-Gallego, N. (2017). Los para-deportes como contenido de educación física en primaria: el ejemplo de la boccia. Didacticae: Revista de Investigación en Didácticas Especificas, 2, 134-142.

Ajzen, I. (1991). The Theory of Planned Behavior. Organizational behavior and human decision processes, 50, 179211. https://doi.org/10.1016/0749-5978(91)90020$\mathrm{T}$

Allport, G. (Ed.) (1954). The nature of prejudice. New York: Doubleday Books.

Bessa, C., Hastie, P., Araujo, A., \& Mesquita, I. (2019).What do we know about the development on personal and social skills within the sport education model:A systematic review. Journal of Sports Science and Medicine, 18(4), 812829.

Bringle, R. G., \& Hatcher, J. A. (1999). Reflection in service learning: Making meaning of experience. Educational Horizons, 77(4), 179-185.

Block, M. E., Haegele, J., Kelly, L., \& Obrusnikova, I. (2020). Exploring Future Research in Adapted Physical Education. Research Quarterly for Exercise and Sport, 1-14. https://doi.org/10.1080/02701367.2020.1741500

Calvo, D., Sotelino, A., \& Rodríguez, J. (2019). AprendizajeServicio e inclusión en educación primaria. Una revisión sistemática desde la Educación Física. Retos, 36, 611-617. https://doi.org/10.47197/retos.v36i36.68972

Capella, C., Gil, J., \& Martí, M. (2014). La metodología del aprendizaje-servicio en la educación física. Apunts. Educación Física y Deportes, 116(2), 33-43. https://doi.org/ 10.5672/apunts.20140983.es.(2014/2). 116.03

Carson, L. R., \& Raguse. L.A. (2014). Systematic Review of Service-Learning inYouth Physical Activity Settings. Quest, 66, 57-95. https://doi.org/10.1080/ 00336297.2013.814578

Chiva-Bartoll, O., \& Fernández-Río, J. (2021). Advocating for Service-Learning as a pedagogical model in Physical Education: towards an activist and transformative approach. Physical Education and Sport Pedagogy. https://doi.org/ 10.1080/17408989.2021.1911981

Chiva-Bartoll, O., Maravé-Vivas, M., Salvador-García, C., \& Valverde-Esteve,T. (2021). Impact of a Physical Education Service-Learning programme on ASD children:A mixed- methods approach. Children andYouth Services Review, 126, 106008. https://doi.org/10.1016/ j.childyouth.2021.106008

Chiva-Bartoll, O., Ruiz-Montero, J., Martín Moya, R., Pérez López, I., Giles Girela, J., García-Suárez, J., \& Rivera-García, E. (2019). University Service-Learning in Physical Education and Sport Sciences: A systematic review. Revista Complutense de Educación, 30(4), 1147-1164. https:// doi.org/10.5209/rced.60191

Evangelio, C., Sierra-Díaz, J., González-Víllora, S., \& Fernández-Río, J. (2018). The sport education model in elementary and secondary education: A systematic review. Movimento, 24(3), 931-946. https://doi.org/10.22456/ 1982-8918.81689

Fernández-Río, J.,Alcalá, D. H., \& Perez-Pueyo, Á. (2018). Revisando los modelos pedagógicos en educación física. Ideas clave para incorporarlos al aula. Revista Española de Educación Física y Deportes, 423, 57-80.

Foley, J.T., Tindall, D., Lieberman, L., \& Kim, S.Y. (2007). How to develop disability awareness using the sport education model. Journal of Physical Education, Recreation \& Dance, 78(9), 32-36. https://doi.org/10.1080/ 07303084.2007 .10598096

García-López, L.M., \& Gutiérrez, D. (2016). Aprendiendo a enseñar deporte. Modelos de enseñanza comprensiva y educación deportiva. Barcelona. INDE.

García-López,L. M., Gutiérrez, D., \& Fernández-Bustos, J. G. (2019). Emprendimiento docente en la transición de educación primaria a educación secundaria: Una propuesta desde la educación deportiva y el aprendizaje servicio. Contextos educativos: Revista de educación, (24), 113-121. http://doi.org/10.18172/con.3913

Guijarro, E., Rocamora, I., Evangelio, C., \& González-Víllora, S. (2020). El modelo de Educación Deportiva en España: una revisión sistemática. Retos, 38, 886-894. https:// doi.org/10.47197/retos.v38i38.77249

González-Víllora, S., Evangelio, C., Sierra-Díaz, J., \& Fernández-Río, J. (2019). Hybridizing pedagogical models: A systematic review. European Physical Education Review, 25(4), 1056-1074. https://doi.org/10.1177/ $1356336 \mathrm{X} 18797363$

Gutiérrez, D., \& Segovia,Y. (2017). Juegos tradicionales, del aula al recreo. Educación Deportiva por Proyectos. Educación Primaria. En O.R. Contreras Jordán \& D. Gutiérrez Díaz del Campo (coords.), El aprendizaje basado en proyectos en Educación Física (pp.39-57) Barcelona: INDE.

Haegele, J.A. (2019). Inclusion Illusion: Questioning the Inclusiveness of Integrated Physical Education. Quest. https://doi.org/10.1080/00336297.2019.1602547

Harvey, S., Kirk, D., \& O'Donovan, T.M. (2014). Sport education as a pedagogical application for ethical 
development in physical education and youth sport. Sport, Education and Society, 19(1), 41-62. https://doi.org/ 10.1080/13573322.2011.624594

Hastie, P.A., Martínez de Ojeda, D., \& Calderón,A. (2011).A review of research on sport education: 2004 to the present. Physical Education and Sport Pedagogy, 16(2), 103-132.https: / /doi.org/10.1080/17408989.2010.535202

Kowalski, E., \& Rizzo, T. (1996). Factors Influencing Preservice Student Attitudes Toward Individuals with Disabilities. Adapted Physical Activity Quarterly, 13(2), 180196. https:/ / doi.org/10.1123/apaq.13.2.180

Menéndez, J.I., \& Fernández-Río, J. (2017). Hybridising sport education and teaching for personal and social responsibility to include students with disabilities. European Journal of Special Needs Education, 32(4), 508-524. https: / /doi.org/10.1080/08856257.2016.1267943

Ministerio de Educación y Formación Profesional (2021). Enseñanzas no universitarias. Alumnado con necesidad específica de apoyo educativo. Curso 2019-2021. Extraído de https://www.educacionyfp.gob.es/dam/ jcr:a3a6c703-ba61-4027-8ee4-bbb0aa2e46ce/ notaresumen20.pdf

Nguyen, D. J., \& Larson, J. B. (2015). Don't forget about the body: Exploring the curricular possibilities of embodied pedagogy. Innovative Higher Education, 40(4), 331-344. http: / / doi.org/10.18172/con.3913

Pérez-Ordás, R., Nuviala,A., Grao-Cruces,A., \& FernándezMartínez, A. (2021). Implementing Service-Learning Programs in Physical Education; Teacher Education as Teaching and Learning Models for All the Agents Involved: A Systematic Review. International journal of environmental research and public health, 18(2), 669. https://doi.org/ 10.3390/ijerph18020669

Pérez-Torralba, A., Reina, R., Pastor-Vicedo, J. C. , \& GonzálezVíllora, S. (2019). Education intervention using parasports for athletes with high support needs to improve attitudes towards students with disabilities in Physical Education. European Journal of Special Needs Education, 34(4), 455-468. https: / / doi.org/10.1080/ 08856257.2018 .1542226

Puig-Rovira, J. M., \& Palos-Rodriguez, J. (2006). Rasgos pedagógicos del aprendizaje- servicio. Cuadernos de Pedagogía, 357, 60-63.

Reina, R., Íñiguez-Santiago, M. C., Ferriz-Morell, R., MartínezGalindo, C., Cebrián-Sánchez, M., \& Roldan,A. (2020). The effects of modifying contact, duration, and teaching strategies in awareness interventions on attitudes towards inclusion in physical education. European Journal of Special Needs Education, 1-17. https://doi.org/10.1080/ 08856257.2020 .1842973

Reina, R., Santana, A., Montesdeoca, R., \& Roldán, A. (2019). Improving Self-
Efficacy Towards Inclusion in In-Service Physical Education Teacher: A Comparison between Insular and Peninsular Region in Spain. Sustainabiliy, 11, 5824. https:/ /doi.org/10.3390/su11205824

Ríos, M. (1994). Los juegos sensibilizadores: Una herramienta de integración social. Revista Apunts, 38, 93-98.

Robles-Rodríguez, J., Abad-Robles, M.T., Fuentes-Guerra, J. G. , \& Benito-Peinado, P. (2017). Los deportes adaptados como contribución a la educación en valores y a la mejora de las habilidades motrices: la opinión de los alumnos de Bachillerato. Retos, (31), 140-144. https://doi.org/ 10.47197/retos.v0i31.49418

Sánchez-Gómez, R. (2011). La enseñanza del Voleibol por niveles de complejidad táctica mediante el enfoque comprensivo. En Modelos actuales de iniciación deportiva: unidades didácticas sobre juegos y deportes de cancha dividida (pp. 153-192). Wanceulen.

Sherrill, C. (2004). Adapted Physical Activity, Recreation and Sport: Crossdisciplinary and Lifespan (6th ed.). New York, NY: McGraw-Hill Companies.

Siedentop, D. (1994). Sport Education: Quality PE though positive sport experiences. Human Kinetics.

Siedentop, D. (2002). Sport education: a retrospective. Journal of Teaching in Physical Education, 21(4), 409-418. https: / / doi.org/10.1123/jtpe.21.4.409

Siedentop. D., Hastie. P., \&Van Der Mars, H. (2020). Complete guide to sport education (3rd ed.). Human Kinetics.

Sparkes,A. C., Martos-Garcia, D. , \& Maher,A. J. (2019). Me, Osteogenesis Imperfecta, and my classmates in physical education lessons: A case study of embodied pedagogy in action. Sport, Education and Society, 24(4), 338-348.https: / /doi.org/10.1080/13573322.2017.1392939

Smith, B. (2008). Imagining being disabled through playing sport: the body and alterity as limits to imagining others' lives. Sport, Ethics and Philosophy, 2 (2), 142-158. https: / / doi.org/10.1080/17511320802222040

Valencia-Peris, A., Mínguez-Alfaro, P., \& Martos-García, D. (2020). La formación inicial del profesorado de Educación Física: una mirada desde la atención a la diversidad. Retos, 37, 597-604. https://doi.org/10.47197/ retos.v37i37.74180

Wallhead, T., \& O’Sullivan, M. (2005). Sport education: Physical education for the new millennium? Physical Education and Sport Pedagogy, 10(2), 181-210. https: / / doi.org/10.1080/17408980500105098

Wilhelmsen, T. , \& Sørensen, M. (2019). Physical educationrelated home-school collaboration: The experiences of parents of children with disabilities. European Physical Education Review, 25(3), 830-846. https://doi.org/ $10.1177 / 1356336 \times 18777263$

Wilson, S., \& Lieberman, L. (2000). DisAbility awareness in physical education. Strategies, 13(6), 12-29. 